\title{
The Implementation of Intelligent Biochemical Monitoring System based on Cloud Platform
}

\section{T.L.Gao}

School of Information Science and Engineering, Shandong Normal University, Jinan, China

Shandong Key Laboratory of Computer Network, Shandong Computer Science Center (National Super

Computing Center in Jinan), Jinan, China

\author{
Y.L.Wang \& M.L.Shu \\ Shandong Key Laboratory of Computer Network, Shandong Computer Science Center (National Super \\ Computing Center in Jinan), Jinan, China
}

\section{S.J.Duan}

School of Information Science and Engineering, Shandong Normal University, Jinan, China

\author{
X.G.Gao \\ School of Science, Shandong Jiaotong University, Jinan, China
}

\begin{abstract}
In order to grasp the developing rules of chronic illness better, give timely and accurate treatment, and reduce the risk of syndromes occuring, we need more time for patients with some of themselves long-term health indicators for monitoring, and adjust the diet structure and treatment. Aiming at the present development situation and social demand of current cloud computing and health awareness, this paper mainly designed a system of Intelligent biochemical monitor system based on cloud computing. The system uses wireless sensor technology, Bluetooth technology, network communication technology and cloud storage technology based on cloud computing. It is composed of the wireless biochemical data collection sensor, intelligent mobile terminal and the cloud platform. It can complete the measurement of biochemical data, wireless data transmission, data storage, historical data trend and intelligent risk early warning function conveniently and reliably.
\end{abstract}

KEYWORD: Wireless intelligent terminal; MSP430; Bluetooth; Biochemistry; Cloud computing

\section{GENERAL INSTRUCTIONS}

With the aging of the population, chronic disease is becoming a growing problem, at the same time the society, families and the economy will be overwhelmed.[1] For patients with chronic diseases such as diabetes, gout, they need regular measurement of their blood glucose, uric acid and other biochemical data, so that they can control the blood glucose, uric acid condition of their own to determine the treatment.[2] Chronic diseases need time measurement, whether at home, unit, leisure places, or on trval. According to the biochemical data of their own to adjust the intake of diet, drugs rationally.[3] Only through the hospital for biochemical data measurement cannot satisfy the demand of monitoring needs, It also need the patients themselves regularly monitor their situation. How to effectively in real time monitoring biochemical data with their own, has become a common concern of the field of health care problems. Aiming at the present development situation and social demand of current cloud computing and health awareness, which is based on cloud computing, build a set of covers perception cloud layer, transport layer, service layer and application layer, including collection terminals, mobile intelligent terminals and cloud ecosystem.

\section{THE OVERALL ARCHITECTURE OF THE BIOCHEMICAL MONITORING SYSTEM BASED ON INTELLIGENT CLOUD PLATFORM}

Intelligent biochemical monitoring system is shown in Figure 1, by wireless biochemical sensor, wireless will monitor the blood glucose, uric acid, user's total cholesterol biochemical data and via Bluetooth transmission to the mobile intelligent terminal. Users can browse the data and historical records at any time through intelligent terminal by themselves. Mobile intelligent terminal will send the monitor data to the cloud platform by Wi-Fi, 2G, 3G, 4G network communication technology. Cloud platform through data mining techniques to analyze historical data, then some feedback information is sent to the mobile terminal, the user can browse their information at any time through the wireless terminal, such as measurement data, the report of 
monitor and treatment recommendations. On the other hand, when there is abnormal measured values outside the normal range, the cloud platform can also send warning information to the user. The user's family also can through the mobile phone, PC, tablet computer and other equipment to get user's data and historical data or other information. The biochemical intelligent monitoring system can not only complete measuring and recording user's health situation timely, but also make users easily understand their condition and the degree of control. The system provides an important data for the adjustment of diet and treatment, and it completes the function to let users long-term condition monitoring. To save the patient's life and property, and prevent the deterioration of the complication, this system also play a role in.

The traditional network consists of three layers system structure, [4] this system based on Internet of things to design intelligent biochemical monitoring system architecture of perception layer, network layer, service layer and application layer of cloud computing.[5] The perceptual layer is using the conventional biochemical detection sensor, with the help of Bluetooth, Wi-Fi, 2G, 3G, 4G network communication technology to complete the network transmission of information. Cloud service layer mainly depend on cloud platform of the National Supercomputer Center in Jinan, and it completes the memory statistics, user's biochemical data of health guidance, monitoring and warning function. Application layer adopts the wireless smart client, responsible for the biochemical data collecting, processing and upload work, also provides a friendly man-machine interactive interface to achieve realtime display of biochemical data.

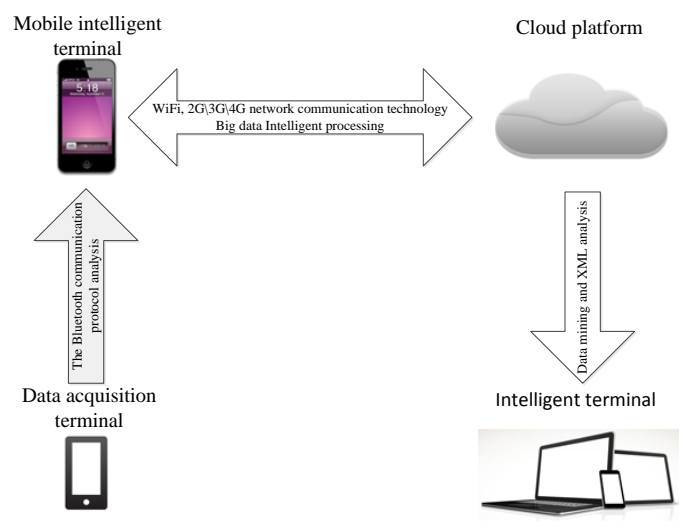

Figure 1 The overall system architecture

\section{THE DESIGN OF THE BIOCHEMICAL MONITORING SYSTEM BASED ON INTELLIGENT CLOUD PLATFORM}

\subsection{Design of collection terminal}

Module design of this system is shown in Figure 2. On the basis of traditional biochemical data collection module, adding the Bluetooth communication module, the biochemical sensor with wireless communication function. It completes the real-time transmission of measurement data, and sends the measuring data or processing data to the wireless intelligent terminal. Bluetooth is a communication technology based on a low-cost short-distance wireless communication.[6] Bluetooth transmission has the advantages of low power consumption and high speed connection.

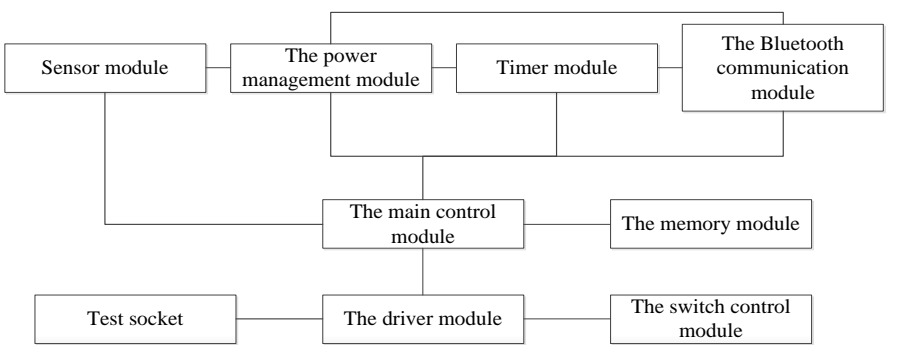

Figure 2 The collection terminal module analysis

Data collection terminal generated signals through sensor enzyme electrical chemical reaction,[7] signal after amplification, filtering, it can calculate the conversion relationship between the biochemical parameters and current voltage characteristic by chemical reaction formula. The AD converter can convert signals into digital signals, the digital signal is sent to the wireless intelligent terminal by the microprocessor through Bluetooth module, and the biochemical data is presented to the display.

The biochemical data collection control unit using MSP430 Series MCU. Transforms the electrical signal of the sensor amplifier filter the high frequency interference, collection and provide high quality signal for subsequent circuit.

\subsection{Design of wireless intelligent terminal client}

The biochemical monitoring system of wireless intelligent terminal client user divided into specific users and related family members or medical personnel, the user can use the client for data display, storage, upload and browse, and user related family members or medical personnel only browse and query data. The combination of these two types of user demand were used to describe the system function.

Combined with sensor data collection, data transmission, display, upload and intelligent processing of the basic flow, system flow of intelligent biochemical monitoring system based on cloud platform is described as follows:

The user tests blood glucose, uric acid, cholesterol and other biochemical data through data collection terminal. Biochemical data is collected automatically through Bluetooth to the wireless intelligent terminal, when the data collection has 
been completed. wireless intelligent terminal uses a friendly human-computer interaction interface to detect the data displayed to the user, and prompt the user whether the current test are normal or not, and appropriate Suggestions are put forward. Key techniques focus on user's detection data privacy and security, stability and accuracy of data transfer protocol, the speed of data transmission. The user uploads biochemical collected data to the cloud platform, then wireless intelligent terminal draws history graph. Cloud platform completes cloud storage of data, technical information for processing the judgment through data mining, and sends the historical average data and basic health advice. When the detection is abnormal data value, it sends text messages warning to avoids the delay in treatment. The user, families and relevant doctor chooses application software or cloud platform to login and get health data through intelligent mobile phone, tablet computer or personal PC timely.

\subsubsection{Upload mechanism of biochemical data on the client}

Because of limited hardware resources mobile phone system, storage capacity and computing power is limited by a lot. When the user needs a large number of data processing, Android application usually cannot meet the requirements. It can only complete the processing of these data on the remote server. In order to complete the Android application on the remote server, we can choose the Web Service.[8] Different platform data has its own characteristics, some platforms are hard to use other platform for data analysis, which formed the "isolated island" phenomenon. Web Services is a kind of thought, a variety of programming languages have their own implementation of it. Application of Web Services to solve the problem of difference between different languages, different platform, using open services in the form of the application program into network applications, and allowing any platform, any location and any language applications to access the service through the internet. Web Services provides a distributed application platform, it makes full use of the loosely coupled distributed programming model of Web, allowing the application of cross platform, multi-language exchange data. All have developed, deployed software, can complete the distributed computing using Web Services.

Cholesterol data, for example, will be collected cholesterol data, user ID and collection date is loaded into the array and written into the database. According to the network transmission protocol of cloud platform, the data according to the specific protocol data frame format is written to a specific location, and then the specified IP address and port number to a upload method, so as to complete the cholesterol upload.

\subsubsection{Draw historical graph}

The need for regular, long-term monitoring for biochemical data to prevent complications caused by the user, a simple, intuitive historical data map is very important. Compared with the traditional way of handwritten notes, this system to display historical data is more visual, systematic, and no handwritten error.

When the user uses the "historical records" query the historical data, the client program to complete the target user data to find from the cloud platform database, to transfer data to a local array, data download shows at the same time, the parameter array passed to the drawing class, complete history graph drawing, as shown in Figure 3:

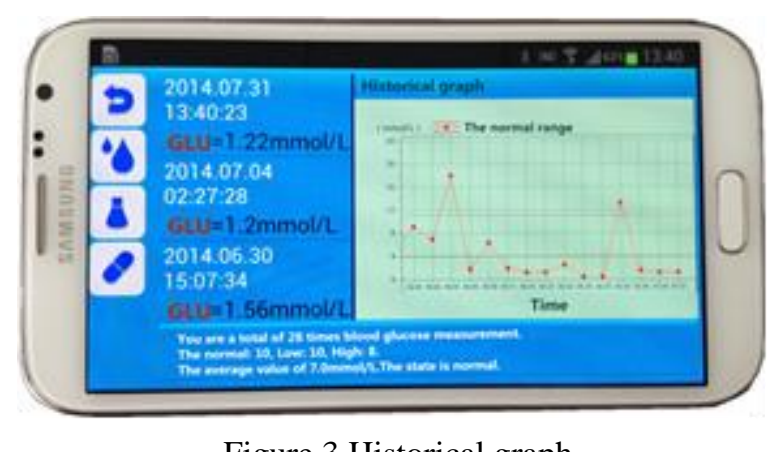

Figure 3 Historical graph

The client program of the system is developed based on the Android platform, inherit the View component in the simple process of drawing, override the onDraw (Canvas canvas) method, the Canvas can draw a lot of graphics. The method uses multiple API drawing, drawing method is provided in the Canvas, and it uses two API, what is a representative of the brush Paint. Another is the representative of any graphics Path. Although this method has a good effect, beautiful appearance, but at the time of the call what has many strict requirements, is very complicated to use. Drawing the system graphics rendering methods used in the introduction of third party of jar packet history curve. The achartengine-0.7.0.jar package packaging a large number of classes and functions can be invoked. In the process of layout, three SurfaceView control planning is built, the left is used for displaying the testing data of the scale value, the control at the blow is used to implement the testing data of the time to date, the rest of the area is used to dynamically display history curve.

By calling the jar XYMultipleSerieDataset package, it builds Dataset of chart and XYSerious. The curve along the $\mathrm{X}$ axis and $\mathrm{Y}$ axis drag effect, and it enables users to clearly see their own historical data. The user can also zoom, drag on the fluctuations in outstanding detail view to a certain extent. For the user to understand the historical data long time segment and health trends, what provides some great convenience. 
The system of cloud service services by the cloud platform of the National Supercomputer Center in Jinan, cloud storage technology which uses the cloud storage technology has changed the past mainly concentrated in the local storage and data processing of the traditional model. Users can conveniently obtain the calculation, storage of required resources, who can find specific data in the huge data and it provides more computing power, more storage space and more reliable services for users.

In the design process of intelligent monitoring system based on cloud platform, considering with the constant improvement of the degree of intelligent wireless terminal, it will increase the demand for data processing, and the lack of wireless terminal data processing capabilities, promotes the necessity of introduction the cloud computing. Cloud computing method bases on data for the task center, storage and management of the use of cluster data resources. In order to guarantee the high speed calculation, high reliability and economic aspects, cloud computing using the redundant storage mode to improve the reliability of the data, using distributed storage technology for data storage to reduce the cost of data processing, using the parallel way to provide services to ensure high speed computing power for the user.

Cloud storage technology has the characteristics of high throughput and high transmission rate characteristics, this system in the face of the large data concurrency, puts forward the intelligent data processing architecture. The architecture includes a main memory database, high-speed distributed storage and high-speed distributed computing. The calculation needs to plan allocation optimization to the data processing node. High speed needs distributed storage to solve the high compression, high speed read and write and high-speed transmission problem. The sign of biochemical data storage and processing principle as shown in Figure4.

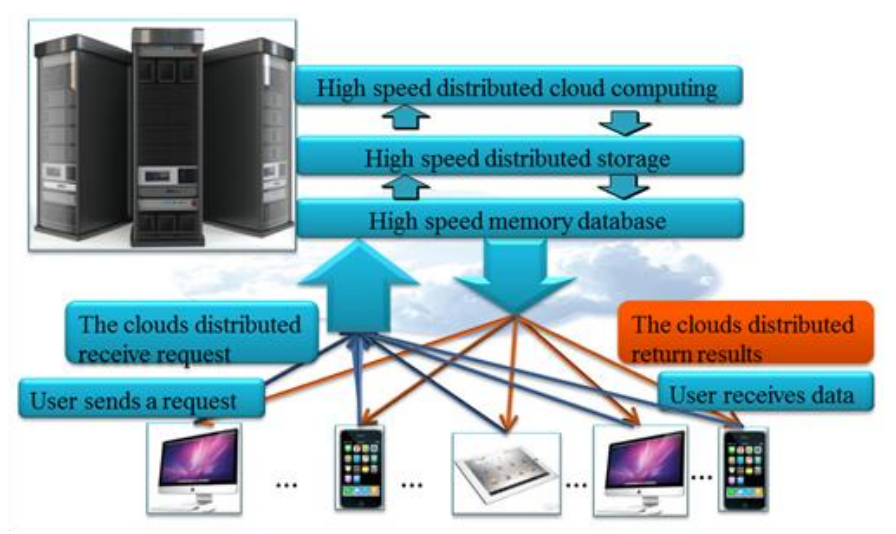

Figure 4 Cloud platform data processing mechanism
Wireless intelligent terminal client operation is simple and convenient, when the user do the blood glucose, uric acid, cholesterol biochemical to test data, they open the biochemical test interface, click on the Bluetooth connection button, wireless intelligent terminal will be automatically connected with the terminal data.

According to the development situation and social demand of current cloud computing and health perception, relying on the cloud computing technology, build a biochemical intelligent monitoring system. The system make the biochemical detection device is easy to carry and get the data, changing from the simple detection to early warning analysis of traditional disease. Through a friendly human-computer interaction interface, the history of the measurement data is displayed and saved. Not only it can display the historical curve data, but also analysis evaluation based on multiple measurement data. Through this approach users and their families can be timely and accurately to learn their biochemical index level. Only in this way, can the users make standard of a good diet and lifestyle.

\section{REFERENCES}

[1] Eman AbuKhousa;Nader Mohamed; Jameela Al-Jaroodi, e-Health Cloud: Opportunities and Challenges, Future Internet, 2012,4(3):621-645

[2] Yibo Zhang, Self monitoring of blood glucose in patients with diabetes mellitus, China Academic Journal Electronic Publishing House, 2006(02)

[3] Hanjing Fu, How to guide the diabetes patients with blood glucose monitoring, Chinese Journal of General Practitioners, 2007(02)

[4] Xu, L.; He, W.; Li, S; Internet of Things in Industries: A Survey, Industrial Informatics, IEEE Transactions on, 2014, PP, No.99:1

[5] Antonio J. Jara; Socrates Varakliotis, Antonio F. Skarmeta; Peter Kirstein, Extending the Internet of Things to the Future Internet through IPv6 support. Mobile Information Systems, 2014,10(1): 3-17

[6] Carles Comez, Joaquim Oller, Josep aradells. Overview and Evaluation of Bluetooth Low Energy: An Emerging Low-Power Wireless Technology. Sensors, 2012, 12: 11734-11753.

[7] Song Xing, Shaofeng Zhang. Design of Portable Blood Glucose Meter Based on Microcontroller MSP430. Measurement \& Control Technology, 2009, 28(2):5-7.

[8] Zhongliang Miao, Xu Zeng, Bin Wan. Further Android application development: core technology analysis and best practice. Beijing: China machine press, 2012: 196-199. 